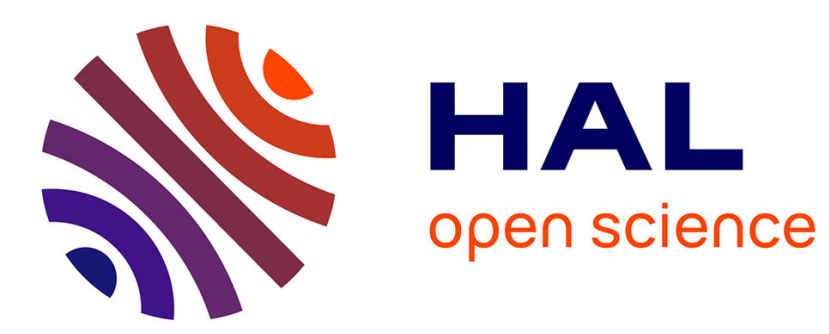

\title{
Perfusion MRI Deconvolution with Delay Estimation and Non-Negativity Constraints
}

Marco Pizzolato, Aurobrata Ghosh, Timothé Boutelier, Rachid Deriche

\section{To cite this version:}

Marco Pizzolato, Aurobrata Ghosh, Timothé Boutelier, Rachid Deriche. Perfusion MRI Deconvolution with Delay Estimation and Non-Negativity Constraints. International Symposium on Biomedical Imaging, Apr 2015, Brooklyn, New York, United States. 10.1109/ISBI.2015.7164057 . hal-01143213

\section{HAL Id: hal-01143213 \\ https://hal.inria.fr/hal-01143213}

Submitted on 16 Apr 2015

HAL is a multi-disciplinary open access archive for the deposit and dissemination of scientific research documents, whether they are published or not. The documents may come from teaching and research institutions in France or abroad, or from public or private research centers.
L'archive ouverte pluridisciplinaire HAL, est destinée au dépôt et à la diffusion de documents scientifiques de niveau recherche, publiés ou non, émanant des établissements d'enseignement et de recherche français ou étrangers, des laboratoires publics ou privés. 


\title{
Perfusion MRI Deconvolution with Delay Estimation and Non-Negativity Constraints
}

\author{
Marco Pizzolato $^{\star} \quad$ Aurobrata Ghosh ${ }^{\star} \quad$ Timothé Boutelier $^{\dagger} \quad$ Rachid Deriche $^{\star}$ \\ * Athena Project-Team, Inria Sophia Antipolis - Méditerranée, France \\ $\dagger$ Olea Medical, La Ciotat, France
}

\begin{abstract}
Perfusion MRI deconvolution aims to recover the timedependent residual amount of indicator (residue function) from the measured arterial and tissue concentration timecurves. The deconvolution is complicated by the presence of a time lag between the measured concentrations. Moreover the residue function must be non-negative and its shape may become non-monotonic due to dispersion phenomena. We introduce Modified Exponential Bases (MEB) to perform deconvolution. The MEB generalize the previously proposed exponential approximation (EA) by taking into account the time lag and introducing non-negativity constraints for the recovered residue function also in the case of non-monotonic dispersed shapes, thus overcoming the limitation due to the non-increasing assumtion of the EA. The deconvolution problem is solved linearly. Quantitative comparisons with the widespread block-circulant Singular Value Decomposition show favorable results in recovering the residue function.
\end{abstract}

Index Terms - Perfusion, Deconvolution, Exponential Bases, Delay, Dispersion, Non-Negative, DSC-MRI

\section{INTRODUCTION}

Perfusion imaging aims to recover parameters related to the passage of blood in the parenchyma (i.e. the functional part) of a tissue. The amount of perfusion is related to the functionality of the parenchyma and its grade of activity.

The perfusion can be characterized in vivo using Dynamic Susceptibility Contrast MRI (DSC-MRI) where a bolus of paramagnetic contrast agent (PA) is injected in the subject's vascular system. In each voxel, a signal related to the PA concentration is acquired on a time sampling grid to derive the correspondent tissue concentration curve $C_{t s}(t)$. According to the indicator-dilution theory [1], the measured $C_{t s}(t)$ in a voxel is expressed as the convolution between the arterial input concentration to the voxel $C_{a}(t)$, and a curve $R(t)$ which expresses at each time the residual amount of PA in the voxel

$$
C_{t s}(t)=\int_{0}^{t} C_{a}(\theta) R(t-\theta) d \theta
$$

The authors express their thanks to Olea Medical and to the ProvenceAlpes-Côte d'Azur Regional Council for providing grant and support. where $R(t)$ is unknown and must be recovered from the observed $C_{a}(t)$ and $C_{t s}(t)$ by means of deconvolution. The quality of the reconstructed $R(t)$ directly affects the estimation of the perfusion parameters such as the blood flow (BF), the blood volume (BV) and the mean transit time (MTT).

Since deconvolution is ill-posed [2] several regularization methods have been proposed to suppress noise, such as the truncated Singular Value Decomposition (tSVD) [3]. More recently a deconvolution method [2] based on exponential bases approximation (EA) showed to outperform tSVD by modeling $R(t)$ as a weighted sum of exponentially decaying functions.

However those techniques show some limitations related to a technical issue inherent the perfusion data acquisition and the physiological nature of the residue function. In real practice, indeed, it is impossible to measure the actual arterial concentration in input to a specific voxel and normally $C_{a}(t)$ is selected as the tissue concentration of a voxel within a region containing only arterial blood. Therefore the concentrations $C_{t s}(t)$ of all the other voxels are physiologically delayed with respect to the selected arterial input $C_{a}(t)$, or can anticipate it, causing the failure of the deconvolution due to the lack of causality assumptions. In addition, the bolus of PA may undergo dispersion which causes the shape of $C_{a}(t)$ to modify, thus changing the natural decreasing shape of $R(t)$ into a non-monotonic one [4]. Such a shape is sometimes considered in myocardial perfusion applications [5]. Moreover, since $R(t)$ expresses the time-dependent remaining quantity of PA in the voxel, negative values are non-physiological and need to be avoided. The EA does not take into account the time lag (delay) between $C_{a}(t)$ and $C_{t s}(t)$. In addition it does not consider dispersion effects since it cannot guarantee non-negativity unless the residue function is constrained to be monotonically decreasing [2].

We propose Modified Exponential Bases (MEB) allowing time delay estimation and a straight forward implementation of non-negativity constraints also in case of non-monotonic dispersed residue functions. We discuss an iterative linear implementation of the MEB and compare the results with the standard block-circulant formulation of tSVD [6], henceforth addressed as oSVD, which is insensitive to the delay and thus comparable with the proposed technique. 


\section{THE DECONVOLUTION PROBLEM}

Assuming the tissue concentration $C_{t s}(t)$ and the arterial one $C_{a}(t)$ both measured on an equally spaced time grid $t_{1}, t_{2}, \ldots, t_{M}$ of size $M$, with $\Delta t=t_{i+1}-t_{i}$, then Eq. 1 is discretized as

$$
C_{t s}\left(t_{j}\right)=\Delta t \sum_{i=0}^{j} C_{a}\left(t_{i}\right) R\left(t_{j}-t_{i}\right)
$$

which can be formulated in matrix form as $\mathbf{c}_{t s}=\mathbf{A r}$, where A is the $M \times M$ convolution matrix containing the samples of the arterial input concentration, $\mathbf{c}_{t s}$ contains the $M$ samples of the tissue concentration and $\mathbf{r}$ contains the $M$ unknown samples of the residue function. If a model $R(t)=G(t, \mathbf{p})$ for the residue function is defined, then the deconvolution problem is finding the set of parameters $\mathbf{p}$. In case of linearity in the parameters the residue function can be written as $\mathbf{r}=\mathbf{G p}$, where $\mathbf{G}$ is the $M \times N$ design matrix and $\mathbf{p}$ the $N \times 1$ vector of coefficients with $N \leq M$. Therefore the convolution is formulated as $\mathbf{c}_{t s}=\mathbf{A G p}$, where $\mathbf{p}$ is unknown.

In [2] it is proposed the exponential approximation (EA) to perform deconvolution, which searches a solution for $R(t)$ in the span of decaying exponential functions, thus modeling the residue function as a sum truncated to an order $N$

$$
R(t)=\sum_{n=1}^{N} k_{n} e^{-\lambda_{n} t} u(t)
$$

where $u(t)$ is the Heaviside step function and the time rates $\lambda_{n}$ are chosen according to the harmonic distribution $\lambda_{n}=n / T$, with $T$ the observation time interval. The unknown parameters $\mathbf{p}=\left[k_{1}, \ldots, k_{N}\right]$ are found with $R(t)$ constrained to be at the same time non-negative $R(t) \geq 0$ and non-increasing $R^{\prime}(t) \leq 0$ for $t>0$, where the non-negativity holds due to the non-increasing assumption [2]. Therefore $\mathrm{EA}$, in order to guarantee non-negativity, neglects dispersion effects assuming a non-increasing shape for $R(t)$. In addition it does not take into account the time delay between $C_{a}(t)$ and $C_{t s}(t)$. In the following section we propose a more general solution solving these limitations.

\section{MODIFIED EXPONENTIAL BASIS}

In order to estimate the time delay $\tau$ and guarantee the non-negativity for both monotonically decreasing and nonmonotonic residue functions, we propose Modified Exponential Bases (MEB). Each basis is constituted as the sum of an exponential decay and the opposite of its derivative with respect to the time rate, respectively weighted by two different constants, $a_{n}$ and $b_{n}$. Each basis shape can thus vary between a pure exponential decay $\left(b_{n}=0\right)$ and its derivative term $\left(a_{n}=0\right)$. Therefore the MEB include the EA and generalize it by integrating the exponential derivative terms. Moreover the time delay $\tau$ is explicitly considered with a Heaviside step function and is included in the exponentials, giving

$$
R(t)=u(t-\tau) \sum_{n=1}^{N}\left(a_{n}+b_{n}(t-\tau)\right) e^{-\alpha_{n}(t-\tau)} .
$$

Hence, we can guarantee non-negativity just by constraining all the $a_{n}$ and $b_{n}$ to be non-negative, and at the same time recover both monotonically decreasing and non-monotonic convolution kernels.

The deconvolution problem incorporating Eq. 4 has a nonlinear solution since the unknown $\alpha_{n}$ and $\tau$ appear in the exponential. However the time rates $\alpha_{n}$ can be preset without loss of generality [2], thus allowing to find an iterative linear procedure to estimate the unknowns.

In order to fix the rates $\alpha_{n}$ we found that the harmonic rule proposed in [2] is too sensitive to the chosen maximum basis order $N$, as also reported in [7]. Despite such a rule can be used, we decide to calculate the rates based on the signal rather than the observation time interval. We define $M T T_{\max }$ as the maximum $M T T$ expected. This can be fixed empirically, or set according to a conservative value calculated from the MTT found with mono-exponential approximation or via oSVD. Then the time rates $\alpha_{n}$ of Eq. 4 are calculated as the reciprocal of the $M T T$ values harmonically distributed in the range $\left[M T T_{\min }, M T T_{\max }\right]$ where $M T T_{\min }=M T T_{\max } / N$.

In order to consider the delay $\tau$ in the estimation we notice that, due to the linearity of the convolution, it can be incorporated in the residue function estimation. To account for this, the problem formulated in Sec. 2 must consider the circular formulation of the convolution [6]. More precisely $C_{t s}(t)$ and $C_{a}(t)$ of length $M$ are extended by zero-padding up to a length $L \geq 2 M$ to avoid aliasing. Then the new $L \times L$ squared circular convolution matrix $\mathbf{A}^{c}$ has entries $A_{i, j}^{c}=C_{a}\left(t_{i-j+1}\right)$ if $j \leq i$ and $A_{i, j}^{c}=C_{a}\left(t_{L+i-j+1}\right)$ if $j>i$. In the rest we drop the superscript for clarity, $A_{i, j}^{c}=A_{i, j}$. The convolution problem is then formulated as $\mathbf{c}_{t s}=\mathbf{A} \mathbf{G}(\tau) \mathbf{p}$, where $\mathbf{G}(\tau)$ is the $L \times 2 N \tau$-dependent design matrix extended to the circular time sampling grid, and $\mathbf{p}$ is the vector containing the $2 \mathrm{~N}$ coefficients $a_{i}$ and $b_{i}$ to be estimated. We perform the estimation of $\tau$ via grid search over a range $\left[\tau_{\min }, \tau_{\max }\right]$ seconds, with a time step $\tau_{s} \leq \Delta t$. The set of estimated parameters $\hat{\mathbf{p}}$ is obtained as the Least Squares solution subject to $a_{i}, b_{i} \geq 0$, $\forall i=1, \ldots, N$ when the estimated delay $\hat{\tau}$ is that minimizing $\left\|\mathbf{c}_{t s}-\mathbf{A G}(\tau) \hat{\mathbf{p}}\right\|_{2}^{2}$ among all $\tau \in\left[\tau_{\min }, \tau_{\max }\right]$.

\section{EXPERIMENTAL DESIGN}

We test the performance of the compared methods with two synthetic datasets, one generated with a decreasing residue function and the other with a non-monotonic (dispersed) one. In the first case we adopt the bi-exponential model $R_{b i-\exp }(t)=A \cdot e^{-\tau_{1} t}+(1-A) \cdot e^{-\tau_{2} t}$, where 


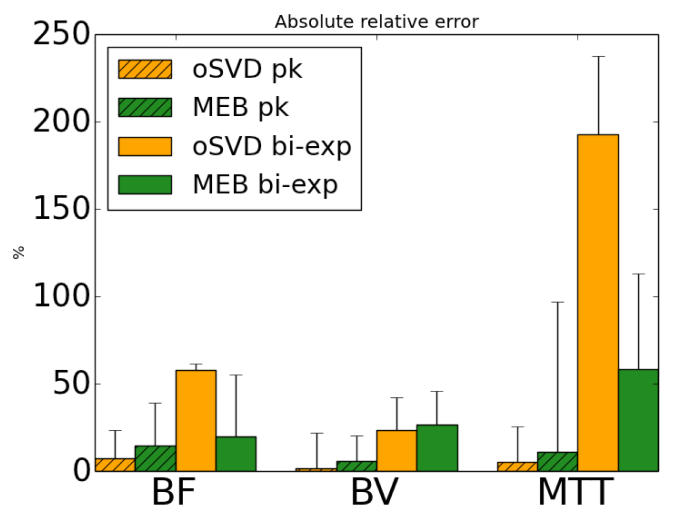

(a) $S N R=80$.

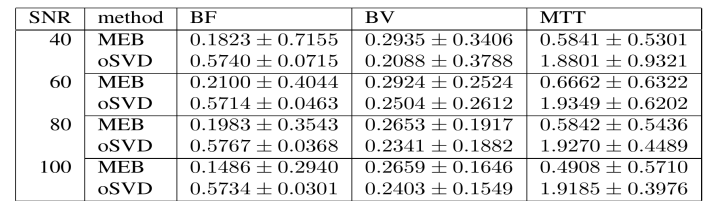

(b) Bi-exponential.

\begin{tabular}{|r|l|l|l|l|}
\hline SNR & method & BF & BV & MTT \\
\hline 40 & MEB & $0.2600 \pm 0.5219$ & $0.0893 \pm 0.2813$ & $0.2011 \pm 0.8228$ \\
& oSVD & $0.2585 \pm 0.2890$ & $0.0370 \pm 0.3681$ & $0.1979 \pm 0.3162$ \\
\cline { 2 - 5 } 60 & MEB & $0.2001 \pm 0.3428$ & $0.0597 \pm 0.1839$ & $0.1609 \pm 0.7685$ \\
& oSVD & $0.1262 \pm 0.1991$ & $0.0186 \pm 0.2496$ & $0.1067 \pm 0.2388$ \\
\cline { 2 - 5 } 80 & MEB & $0.1460 \pm 0.2434$ & $0.0558 \pm 0.1497$ & $0.1112 \pm 0.8578$ \\
& oSVD & $0.0738 \pm 0.1609$ & $0.0172 \pm 0.1998$ & $0.0531 \pm 0.2031$ \\
\cline { 2 - 5 } 100 & MEB & $0.1130 \pm 0.1942$ & $0.0353 \pm 0.1169$ & $0.0938 \pm 0.8429$ \\
& oSVD & $0.0522 \pm 0.1253$ & $0.0139 \pm 0.1559$ & $0.0515 \pm 0.1696$ \\
\hline
\end{tabular}

(c) Pharmacokinetic.

Fig. 1. Absolute relative error of estimates (mean and standard deviation).

$\tau_{1}=0.68, \tau_{2}=0.05$ and $A=0.95$, according to the normal tissue values given in [8]. In the second case, we use the Pharmacokinetic model as formulated in [7], $R_{p k}(t)=$ $\left(e^{-\lambda_{1} t}-e^{-\lambda_{2} t}\right) /\left(\lambda_{2}-\lambda_{1}\right)-\left(e^{-\lambda_{1} t}-e^{-\lambda_{3} t}\right) /\left(\lambda_{3}-\lambda_{1}\right)$ where we fix $\lambda_{2}=0.21, \lambda_{3}=0.36$ according to [5] and $\lambda_{1}=\left(\lambda_{3}-\lambda_{2}\right) /\left(M T T \cdot \lambda_{2} \cdot \lambda_{3}\right)$ with $M T T \approx 2.2 \mathrm{~s}$, as obtained from the bi-exponential model. We generate the actual residue function via multiplication of $R_{b i-\exp }(t)$ or $R_{p k}(t)$ with $B F=30 \mathrm{ml} / 100 \mathrm{~g} / \mathrm{min}$, giving peak values of $B F$ for the bi-exponential model and $\approx 5.7 \mathrm{ml} / 100 \mathrm{~g} / \mathrm{min}$ for the pharmacokinetic one. In the latter case, the peak value will be considered as $B F$ for the experiments. With the chosen parametrization, $B V \approx 1.1 \mathrm{ml} / 100 \mathrm{~g}$ in all cases. The arterial concentration is generated according to [4] as $C_{a}(t)=\gamma_{0}\left(t-t_{0}\right)^{\nu} \cdot e^{-\left(t-t_{0}\right) / \beta}$ with $\nu=3, \beta=1.5, \gamma_{0}=1$, and $t_{0}=30 \mathrm{~s}$. The tissue concentration $C_{t s}(t)$ is then obtained via Eq. 2. The concentration curves are then converted to signal intensities according to $S(t)=S_{0} \cdot e^{-\kappa \cdot C(t) \cdot T E}$, where $T E$ is the echo time and $\kappa$ is the susceptibility constant fixed according to [3]. We choose $S_{0}$ and $T E$ separately for the tissue concentration $S_{0}^{t}=200, T E_{t}=55 \mathrm{~ms}$ and for the arterial one $S_{0}^{a}=600, T E_{a}=13 \mathrm{~ms}$, according to [9]. The signals, obtained with repetition time $\Delta t=1 \mathrm{~s}$, are then corrupted with Gaussian noise with zero mean and standard deviation $\sigma=S_{0} / S N R$, and finally reconverted in concentration curves.

We performed the experiments separately for $C_{t s}(t)$ obtained after convolution of $C_{a}(t)$ with both the bi-exponential and the pharmacokinetic kernel. In each case we delayed $C_{a}(t)$ and $C_{t s}(t)$ with integer values in range $[-5,5]$ seconds. For each delay we calculated the residue function via $\operatorname{oSVD}(20 \%)$ and MEB $\left(N=30\right.$, harmonic $\alpha_{n}, M T T_{\max }=$ $\left.4 \cdot M T T_{o S V D}, \tau_{\min }=-10 \mathrm{~s}, \tau_{\max }=15 \mathrm{~s}, \tau_{\mathrm{s}}=0.25 \mathrm{~s}\right)$ deconvolution, and obtained the estimates of $B F, B V$ and $M T T$. We tested four $S N R$ values [9], [40, 60, 80, 100], generating 100 noisy repetitions for each possible combination of kernel, delay and $S N R$.

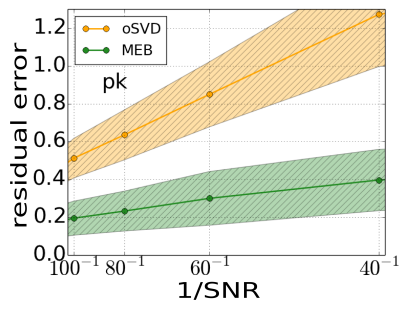

(a) Pharmacokinetic kernel

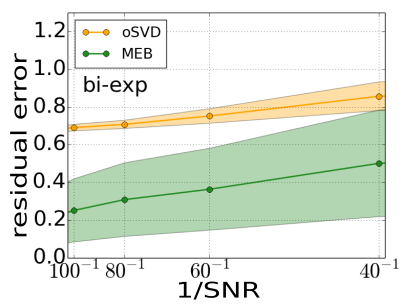

(c) Bi-exponential kernel

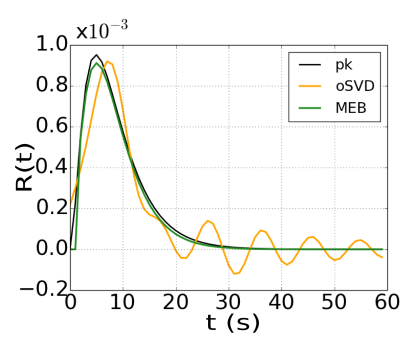

(b) fitting, $S N R=80$

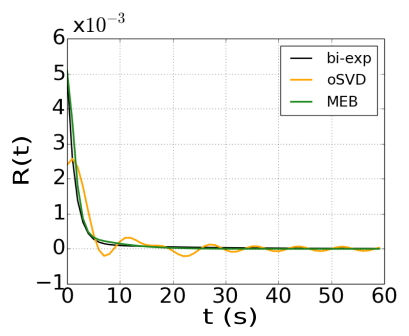

(d) fitting, $S N R=80$
Fig. 2. Residual error $(a, c)$ and residue function fitting $(b, d)$.

\section{RESULTS AND DISCUSSION}

Figure 1(a) illustrates the mean absolute relative error in the estimation of each parameter for the representative case of $S N R=80$. Figures $1(b)$ and $1(c)$ show the tables giving the estimates mean errors and standard deviations for all the $S N R$, in the case of bi-exponential and pharmacokinetic kernel respectively. For both oSVD and MEB, we computed the mean residual error of the recovered residue function with respect to the ground truth, as shown in Fig. 2(a) and 2(c), for increasing noise levels, in case of dispersed and the bi-exponential kernel respectively; the std areas are shown. Fig. 2(b) and 2(d) show examples of the correspondent MEB and OSVD fittings against the ground truth. 
We notice that, for both MEB and oSVD, the quality of the estimated parameters depends on the chosen convolution kernel (Fig. 1). In the case of a smooth kernel, such as the pharmacokinetic one, the MEB show less effective results than oSVD in estimating the parameters (table. 1(c)). Indeed oSVD cuts the high frequency oscillations of $C_{a}(t)$ which, in such a case, mainly correspond to noise. A possible explanation of the MEB performance is that the delay estimation is harder on the dispersed kernel due to its smoothness. Indeed, we found that the delay estimation error for the pharmacokinetic kernel doubles that obtained for the bi-exponential one $(\approx 42 \%$ against $\approx 19 \%, S N R=80)$.

On the other hand oSVD show major limitations in the case of bi-exponential residue function. For instance the estimation error of $M T T$ almost doubles the magnitude of the correspondent ground truth value. This performance drop of oSVD is highly related to the marked underestimation of $B F$, with an error around the $57 \%$ of the actual blood flow as shown in Fig. 1(a) and in table 1(b).

The MEB outperform oSVD for the bi-exponential residue function as shown in Fig. 1(a) and in table 1(b). Indeed the MEB manage to reduce the underestimation of $B F$ giving an average relative error in a range $[15 \%, 21 \%]$ of the ground truth value. The MEB also allow to obtain an error for $M T T$ always at least three times smaller than that of oSVD. The calculation of $B V$ as the analytical integral of $R(t)$ obtained via MEB is comparable to that obtained via oSVD. However it is normally preferable to estimate $B V$ directly from the concentration curves $C_{a}(t)$ and $C_{t s}(t)$ to avoid introducing errors related to the deconvolution procedure.

The MEB show less residual error then oSVD for both of the tested kernels and at any $S N R$, as shown in Fig. 2(a) for the dispersed residue function and $2(c)$ for the bi-exponential one. We further notice that the MEB fitting is more robust to noise. Indeed Fig. 2(a) shows that with dispersed residue function, the difference between the oSVD residual error at $S N R=40$ and $S N R=100$ is almost four times higher than the correspondent difference for the MEB. The fitting capability of the MEB is more marked for the bi-exponential kernel, Fig. 2(c), where the amount of residual error stands between the $35 \%$ and the $60 \%$ of the oSVD error for any $S N R$. In general, the residue function recovered via MEB looks smoother and without ripples, which are a contributing cause to the oSVD residual error.

\section{CONCLUSIONS}

We introduced Modified Exponential Bases to perform perfusion deconvolution. The proposed MEB generalize the exponential approximation by including both exponentially decaying terms and their derivatives. Indeed the MEB allow to take into account residue functions which are either purely decreasing or dispersed, providing at the same time a straight forward implementation of non-negativity constraints. The
MEB also explicitly take into account the time delay between the arterial and the tissue concentrations. The oSVD showed a lower error in the perfusion parameters estimation for the dispersed residue function, in agreement with the latter's smoothness. However, in the case of the bi-exponential kernel the MEB outperform oSVD. In addition, for any type of kernel, the fitting results of the MEB compare favorably with respect to those obtained via oSVD, and are more robust to noise. We believe that time delay estimation and non-negativity constraints help in recovering a physiologically meaningful residue function. Moreover we find that performing deconvolution with continuous basis functions improves the robustness to noise and we think that proposed bases should take into account the variability in the possible shapes of the residue function.

\section{REFERENCES}

[1] Meier et al., "On the theory of the indicator-dilution method for measurement of blood flow and volume," $J$ Appl Physiol, vol. 6(12), pp. 731-744, 1954.

[2] Keeling et al., "Deconvolution for dce-mri using an exponential approximation basis," Med Image Anal, vol. 13(1), pp. 80-90, 2009.

[3] Østergaard et al., "High resolution measurement of cerebral blood flow using intravascular tracer bolus passages. part i: Mathematical approach and statistical analysis," Magn Reson Med, vol. 36(5), pp. 715-725, 1996.

[4] Calamante et al., "Delay and dispersion effects in dynamic susceptibility contrast mri: simulations using singular value decomposition," Magn Reson Med, vol. 44(3), pp. 466-473, 2000.

[5] Zarinabad et al., "Voxelwise quantification of myocardial perfusion by cardiac magnetic resonance," Magn Reson Med, pp. 1994-2004, 2012.

[6] Wu et al., "Tracer arrival timinginsensitive technique for estimating flow in $\mathrm{mr}$ perfusionweighted imaging using singular value decomposition with a blockcirculant deconvolution matrix," Magn Reson Med, vol. 50(1), pp. 164-174, 2003.

[7] Batchelor et al., "Arma regularization of cardiac perfusion modeling," ICASSP, pp. 642-645, March 2010.

[8] Mehndiratta et al., "Modeling the residue function in dscmri simulations: Analytical approximation to in vivo data," Magn Reson Med, 2013.

[9] Wirestam et al., "Wavelet-based noise reduction for improved deconvolution of time-series data in dynamic susceptibility-contrast mri," Magn Reson Mater Phy, vol. 18(3), pp. 113-118, 2005. 